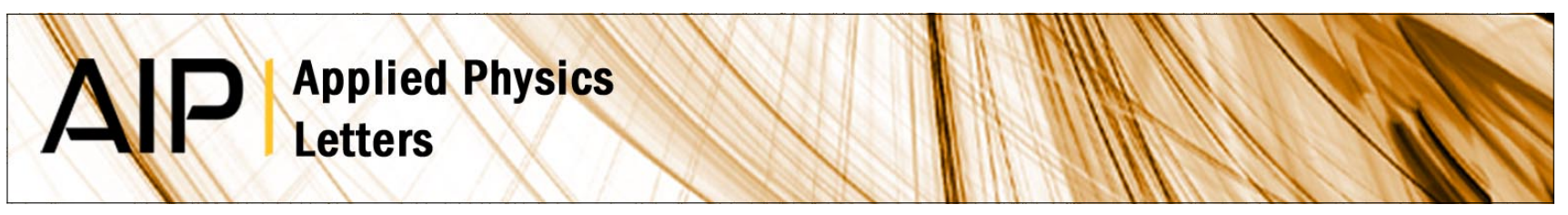

\title{
Optical monitoring of InP monolayer growth rates
}

P. J. Parbrook, K. B. Ozanyan, M. Hopkinson, C. R. Whitehouse, Z. Sobiesierski et al.

Citation: Appl. Phys. Lett. 73, 345 (1998); doi: 10.1063/1.121829

View online: http://dx.doi.org/10.1063/1.121829

View Table of Contents: http://apl.aip.org/resource/1/APPLAB/v73/i3

Published by the American Institute of Physics.

\section{Related Articles}

Spontaneous emission control of single quantum dots in bottom-up nanowire waveguides Appl. Phys. Lett. 100, 121106 (2012)

Europium location in the AIN: Eu green phosphor prepared by a gas-reduction-nitridation route J. Appl. Phys. 111, 053534 (2012)

Bi-induced p-type conductivity in nominally undoped $\mathrm{Ga}(\mathrm{AsBi})$

Appl. Phys. Lett. 100, 092109 (2012)

Enhancement of light extraction efficiency by evanescent wave coupling effect in ridge-shaped AIGalnP/GalnP quantum wells

Appl. Phys. Lett. 100, 091107 (2012)

Compositional instability in InAIN/GaN lattice-matched epitaxy

Appl. Phys. Lett. 100, 092101 (2012)

\section{Additional information on Appl. Phys. Lett.}

Journal Homepage: http://apl.aip.org/

Journal Information: http://apl.aip.org/about/about_the_journal

Top downloads: http://apl.aip.org/features/most_downloaded

Information for Authors: http://apl.aip.org/authors

\section{ADVERTISEMENT}

\section{(1) ACCELERATE AMBER AND NAMD BY $5 X$. NVIDIA TRYIT ONA FREE, REMOTELYYHOSTED CLUSTER.}




\title{
Optical monitoring of InP monolayer growth rates
}

\author{
P. J. Parbrook, ${ }^{\text {a) }}$ K. B. Ozanyan, M. Hopkinson, ${ }^{\text {b) }}$ and C. R. Whitehouse \\ IRC for Semiconductor Materials, Department of Electronic and Electrical Engineering, \\ University of Sheffield, Sheffield, S1 3JD, United Kingdom
}

Z. Sobiesierski and D. I. Westwood

Department of Physics and Astronomy, University of Wales Cardiff, Cardiff, CF2 3YB, United Kingdom

(Received 10 November 1997; accepted for publication 13 May 1998)

\begin{abstract}
Reflection anisotropy (RA) spectroscopy has been used to examine the in vacuo (001) surface of InP before and during growth by molecular beam epitaxy (MBE). The dominant effect on the RA signal occurring the initiation of growth is the change in the surface V/III ratio, caused by the exposure of the surface to the incident indium flux. During MBE growth of InP under commonly used conditions, RA oscillations are clearly observed. These oscillations have been confirmed to correspond to the growth of InP monolayers. The oscillations are tentatively ascribed to the variation in $\mathrm{P}$ coverage during the growth of each monolayer of material. (C) 1998 American Institute of Physics. [S0003-6951(98)01129-2]
\end{abstract}

As the design criteria for semiconductor devices become progressively more stringent, there has been increasing interest in methods for in situ monitoring of the epitaxial growth of semiconductor layers. Techniques that can probe the properties of the growing epitaxial material are particularly attractive. In molecular beam epitaxy (MBE), reflection highenergy electron diffraction (RHEED) is used extensively to examine both the surface structure and also to monitor layer growth rates, via the observed oscillations of the specular spot intensity as each monolayer is grown. ${ }^{1}$ However, for metalorganic vapor phase epitaxy (MOVPE), it is not possible to use such electron probes due the relatively high pressure growth ambient. In this case surface structure measurements have been successfully carried out using grazing incidence $\mathrm{x}$-ray scattering, ${ }^{2}$ but this technique requires an intense $\mathrm{x}$-ray source, only achievable using a synchrotron.

Optical probes can, in principle, be applied in either the MBE or MOVPE growth environment. For example, measurement of the bulk properties of a growing epilayer can be made using reflectometry ${ }^{3}$ or ellipsometry. ${ }^{4}$ However, in order to study semiconductor growth processes, more complex, surface-sensitive techniques using polarized light have been developed, including reflection anisotropy spectroscopy $(\mathrm{RAS})^{5}$ and surface photoabsorption. ${ }^{6}$ These latter techniques can provide chemical information about the surface, complementing the structural information that RHEED can provide. However, to date, most reported studies of semiconductor surfaces using optical probes have concentrated on (001) GaAs, with only a few studies relating to InP. Comparison of (001) InP surfaces produced by chemical beam epitaxy (CBE) with those produced by MOVPE has been performed by Zorn et al., ${ }^{7}$ and the dynamics of InP growth by MOVPE has been examined by Knorr et al. ${ }^{8}$ In addition, Dietz et $a .^{9}{ }^{9}$ have examined the growing InP surface, using atomic layer epitaxy (ALE) conditions in a CBE reactor. However, there are few studies using RAS as a probe, of the

\footnotetext{
${ }^{a)}$ Electronic mail: p.parbrook@ sheffield.ac.uk

${ }^{b}$ Also at EPSRC Central Facility for III-V Semiconductors.
}

growth of InP by solid-source MBE. Recently, we have examined the InP surface, prepared by MBE, using a combination of RHEED and RAS, ${ }^{10}$ leading to the production of a detailed surface phase diagram for the (001) InP surface under static, no-growth, conditions. In this letter, the "dynamic" features observed during InP MBE growth are reported.

InP growth was carried out in a VG Semicon V80H MBE machine, equipped with elemental $\mathrm{In}$ and $\mathrm{P}_{2}$ dimer sources. After oxide desorption at $520-530{ }^{\circ} \mathrm{C}$ under a $\mathrm{P}_{2}$ overpressure, a thin, $0.2 \mu \mathrm{m}$, homoepitaxial InP buffer was grown on the (001) substrate using a substrate temperature of $510{ }^{\circ} \mathrm{C}$, calibrated using pyrometer measurements. Subsequent InP growth was carried out under a wide range of conditions: the substrate temperature was varied between 410 and $540{ }^{\circ} \mathrm{C}$, the $\mathrm{P}_{2}$ beam equivalent pressure was varied between $1.2 \times 10^{-6}$ and $5.2 \times 10^{-6} \mathrm{mbar}$, and the In source temperature was varied to give growth rates between 0.34 and 0.81 monolayers per second $\left(\mathrm{MLs}^{-1}\right)$.

RHEED was used to estimate the growth rate by monitoring the variation in intensity of the specular (100) reflection, it was also used to confirm the InP surface reconstruction before, during, and after growth. Reflection anisotropy (RA) spectra were recorded, in the range from 1.5 to $5.5 \mathrm{eV}$, using a conventional spectrometer of the type developed by Aspnes et al. ${ }^{5}$ Optical access was made to the sample using a strain-free window mounted on the pyrometer viewport, situated at normal incidence to the sample surface. The real part of the variation of the RAS signal, which has a much reduced sensitivity to window strain, was recorded. The results were taken either as RA spectra under time-integrated conditions, or as changes in the RA signal at various preselected photon energies as a function of time.

The reconstructions observed for the static (001) InP surface, across a range of substrate temperature and phosphorus overpressure, have been examined by RHEED and RAS. ${ }^{10}$ For temperatures above $460{ }^{\circ} \mathrm{C}$, at a constant $\mathrm{P}_{2} \mathrm{BEP}$ of $3.5 \times 10^{-6}$ mbar, RHEED indicates a $(2 \times 4)$ reconstruction. However, RAS indicates that there are actually two stable 


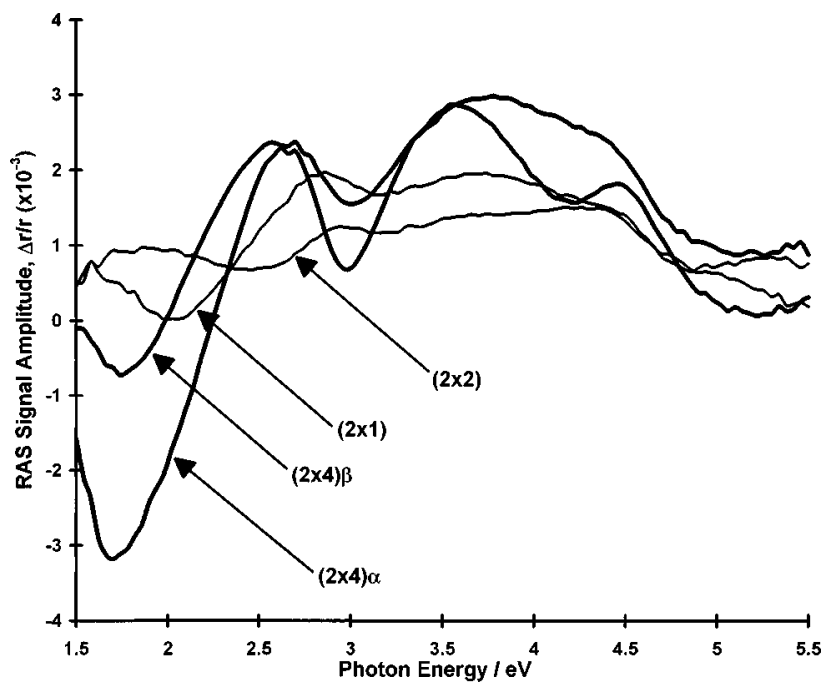

FIG. 1. RA spectra recorded from the (001) InP surface at substrate temperatures corresponding to different RHEED reconstructions, under a constant $\mathrm{P}_{2}$ BEP of $3.5 \times 10^{-6}$ mbar. The $(2 \times 4) \alpha$ and $\beta$ reconstructions are of most relevance to the standard MBE growth conditions used for InP, and are indicated by the thick lines.

( $2 \times 4)$ reconstructed surfaces in this regime, identified as $\alpha$ and $\beta$, depending on whether the substrate temperature is above or below $510{ }^{\circ} \mathrm{C}$, respectively. RHEED indicates that the surface reconstruction changes to $(2 \times 1)$ for substrate temperatures between 460 and $420^{\circ} \mathrm{C}$, and at lower temperatures a $(2 \times 2)$ reconstruction is observed. Figure 1 shows typical RAS recorded for each indicated RHEED reconstruction.

Homoepitaxial MBE growth of InP usually takes place at a substrate temperature in the region of $480{ }^{\circ} \mathrm{C},{ }^{11}$ which, under static conditions, corresponds to a $(2 \times 4) \beta$ reconstruction. Figure 2 illustrates the observed spectral dependence of the change in the RA signal of a $(2 \times 4) \beta$ static surface caused by (i) opening the indium cell shutter to grow an InP layer at $0.5 \mathrm{MLs}^{-1}$, (ii) increasing the substrate temperature by $20^{\circ} \mathrm{C}$, and (iii) reducing the $\mathrm{P}_{2}$ BEP from 1.15 $\times 10^{-6}$ to $3.5 \times 10^{-7}$ mbar is shown. In each case, the observed spectral change is seen to be virtually identical, mov-

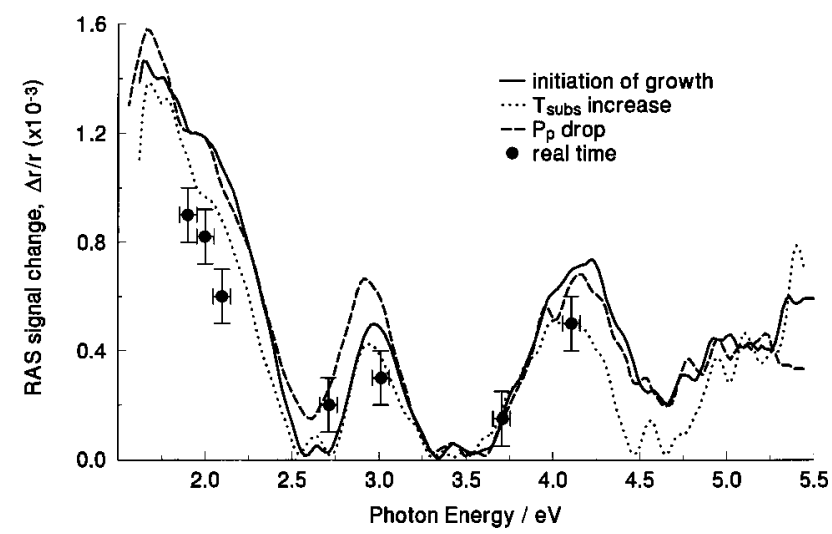

FIG. 2. The change in RA spectra from a "standard" $(2 \times 4) \beta$ reconstructed InP surface recorded for (i) initiation of InP growth (at $0.5 \mathrm{MLs}^{-1}$ ), (ii) a $20^{\circ} \mathrm{C}$ increase in substrate temperature, and (iii) a reduction in $\mathrm{P}_{2}$ flux of $8 \times 10^{-7}$ mbar. The dots represent the RA signal change observed at the moment of growth initiation, recorded during time scans at a constant photon energy for an InP growth rate of $0.5 \mathrm{MLs}^{-1}$.

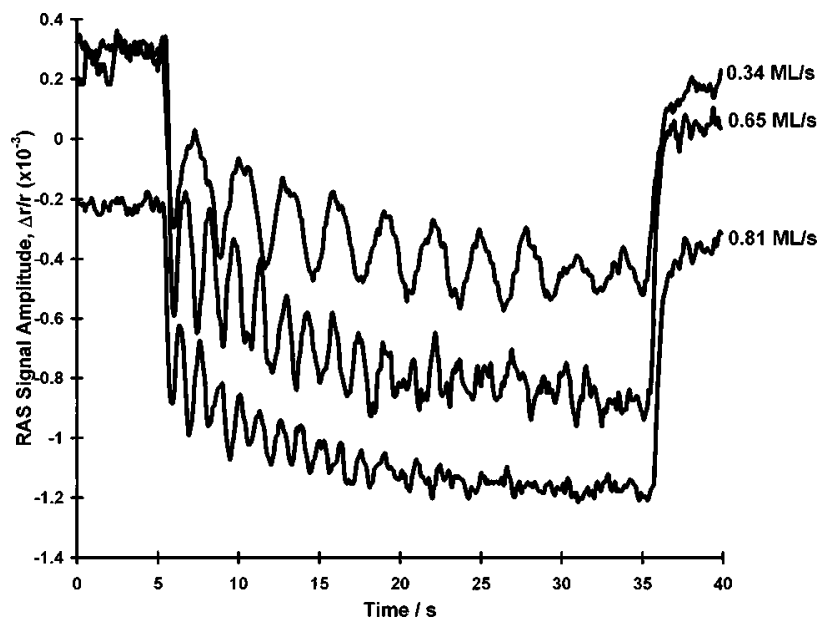

FIG. 3. Time-dependent changes in the observed RA signal at $2.0 \mathrm{eV}$, recorded on the initiation of InP growth at the indicated rates.

ing the RA signal towards that expected for a $(2 \times 4) \alpha$ surface. The magnitude of the observed deviation is particularly large around $1.7 \mathrm{eV}$, with smaller deviations at around 3 and $4.2 \mathrm{eV}$. The dots in Fig. 2 indicate the magnitude of the jump in the RA signal observed at fixed photon energies on opening of the indium shutter (i.e., initiating InP growth). The substrate temperature, $\mathrm{P}_{2} \mathrm{BEP}$ and In flux correspond to those used to produce curve (i), and the jumps observed lie close to those expected from this curve. The observed signal transient times are close to the lock-in integration time constant and comparable to the monolayer growth times.

The time-dependent scans also show clear oscillations in the RA signal during the first seconds of growth, the strength of which are approximately in proportion to the size of the initial signal change occurring on the initiation of growth. These oscillations are observed to have a period identical to that of the corresponding RHEED oscillations recorded under identical conditions. The oscillations observed at photon energies close to the spectral feature at $1.7 \mathrm{eV}$ were found to be the strongest and most easily resolved over the widest range of growth conditions. However, due to the decaying intensity of the light source at these low photon energies which leads to poor signal-to-noise ratios, a photon energy of $2.0 \mathrm{eV}$ was selected for subsequent experiments performed to examine the variation in RA oscillation intensity versus both substrate temperature and $\mathrm{P}_{2}$ overpressure.

The oscillations are clearest for substrate temperatures between 470 and $490{ }^{\circ} \mathrm{C}$. Outside this window the oscillation strength decays gradually, becoming irresolvable at temperatures below 440 or above $530^{\circ} \mathrm{C}$. The oscillations became stronger and damped more slowly for increasing $\mathrm{P}_{2}$ overpressures, up to the highest BEP examined of 5.2 $\times 10^{-6}$ mbar. For the lowest $\mathrm{P}_{2}$ BEP examined of 1.2 $\times 10^{-6}$ mbar the oscillations damped very quickly, unless the growth rate was lowered to $0.34 \mathrm{MLs}^{-1}$. Under such conditions the optimum substrate temperature to observe of oscillations was found to drop to around $440{ }^{\circ} \mathrm{C}$.

As an example, Fig. 3 shows the oscillations observed at $2.0 \mathrm{eV}$, for a $\mathrm{P}_{2} \mathrm{BEP}$ of $5.2 \times 10^{-6} \mathrm{mbar}$ and a substrate temperature of $470{ }^{\circ} \mathrm{C}$, for three different $\mathrm{InP}$ growth rates. In all cases, clear oscillations were observed for at least nine periods. For the slowest growth rate examined 
$\left(0.34 \mathrm{MLs}^{-1}\right)$, up to 14 cycles could be recorded without using any data-smoothing techniques.

The identical periodicity of the recorded RHEED and RA oscillations shows that the RA signal changes are due to the deposition of InP monolayers. Such RA oscillations have been observed previously for the growth of GaAs, both by $\mathrm{MBE}^{12}$ and MOVPE, ${ }^{13}$ but apart from the report by Dietz et al., ${ }^{9}$ where oscillations are reported under ALE-like conditions, no such reports have been published for InP growth. The monolayer RA oscillations observed for GaAs grown by MBE are generally weak, and damp quickly. ${ }^{12}$ In contrast, the RA oscillations observed here for InP are strong, and easily recorded at conventional MBE growth rates of close to $1 \mathrm{MLs}^{-1}$, whilst even stronger oscillations are detected at lower growth rates. The intensity of these oscillations is optimal at the typical substrate temperature used for InP growth of around $480^{\circ} \mathrm{C}$.

An examination of Fig. 1 shows that there are a number of RA spectral features associated with the $(2 \times 4) \alpha$ and $(2 \times 4) \beta$ reconstructions. In both cases, there is a peak around $2.7 \mathrm{eV}$, which is generally associated with P dimerized along the $[\overline{1} 10]$ direction. ${ }^{10}$ This feature changes only slightly during the transition between the two reconstructions. At $1.7 \mathrm{eV}$, there is a strong dip in the RA signal for the $(2 \times 4) \alpha$ surface which is suppressed in the $(2 \times 4) \beta$ case. By analogy with GaAs, this feature has been ascribed to In dimerized along the [110] direction. ${ }^{10}$ While the features at higher photon energy are less well understood, a key difference between the two RA signals is the intensity of the feature reflecting the surface density of In dimers.

In Fig. 2, it was shown that the observed spectral deviations are virtually identical when increasing substrate temperature, reducing $\mathrm{P}_{2}$ overpressure or initiating growth. With increasing substrate temperature, the phosphorus coverage on the surface drops due to enhanced $\mathrm{P}$ desorption, and this relative P:In coverage will also drop on the initiation of growth, due to the incident In flux. The RA signal transients on the initiation of growth also lie close to these curves for the spectral deviation, providing further evidence that the change in phosphorus coverage is the dominant effect governing the RA spectral shifts observed on starting growth.

RA oscillations observed during the growth of GaAs, both by MBE and MOVPE, have been examined recently by Zettler et al. ${ }^{14}$ These authors point out that, for the growth of GaAs by MBE, the variation in step-edge density during the growth of a monolayer can cause a variation in the As coverage of the surface due to the reduced As coverage around surface steps, as observed by Payne et al. ${ }^{15}$ Such a variation in group $\mathrm{V}$ coverage can then lead to significant changes in the RA signal amplitude, particularly in when the surface conditions are close to the boundary between two surface reconstructions. It should be noted that the growth conditions for both GaAs and InP are different for MBE and MOVPE and that consequently, as Zettler et al. ${ }^{14}$ detail for the GaAs case, the growth mechanism and RAS results must be dealt with on a case-by-case basis.
The RA oscillations observed in these experiments have been tentatively ascribed to RA signal changes corresponding to variations in $\mathrm{P}$ coverage on the InP surface. Following the GaAs model, ${ }^{14}$ this change in $\mathrm{P}$ coverage is induced by cyclic variation in surface step density during the growth of each monolayer of material. At a substrate temperature of around $480^{\circ} \mathrm{C}$, the RA oscillation intensity is particularly strong, as the initiation of growth causes a significant increase in the number of In dimers as the surface changes to become more $(2 \times 4) \alpha$ like in nature. The variation in surface step density during the deposition of each monolayer then causes a change in the density of In dimers on the surface, which can be monitored by the RA signal changes in the $1.5-2.0 \mathrm{eV}$ spectral region.

In conclusion, we have observed oscillations in the RA signal during the MBE growth of (001) InP. These RA oscillations are observed in the range of substrate temperature and $\mathrm{P}_{2}$ BEP conventionally used for InP growth, and for practical growth rates of around $1 \mathrm{MLs}^{-1}$. The oscillations are ascribed to the variation in the $\mathrm{P}$ coverage of the surface due to the cyclic change in surface step density during each ML of growth. The clarity of the oscillations observed provide a clear indication of the potential of RAS as an in situ real-time growth monitoring technique.

${ }^{1}$ R. Ludeke, R. M. King, and E. H. C. Parker, The Technology and Physics of Molecular Beam Epitaxy, edited by E. H. C. Parker (Plenum, New York, 1985)

${ }^{2}$ P. H. Fuoss, D. W. Kisker, G. B. Stephenson, and S. Brennan, Mater. Sci. Eng., B 30, 99 (1995).

${ }^{3}$ T. Farrell, J. V. Armstrong, and P. Kightley, Appl. Phys. Lett. 59, 1203 (1991).

${ }^{4}$ D. E. Aspnes, Thin Solid Films 233, 1 (1993).

${ }^{5}$ D. E. Aspnes, J. P. Harbison, A. A. Studna, and L. T. Florez, J. Vac. Sci. Technol. A 6, 1327 (1988).

${ }^{6}$ N. Kobayashi, T. Makimoto, Y. Yamauchi, and Y. Horikoshi, J. Cryst. Growth 107, 62 (1991).

${ }^{7}$ M. Zorn, T. Trepk, J.-T. Zettler, C. Meyne, K. Knorr, Th. Wethkamp, W. Richter, B. Junno, M. Miller, and L. Samuelson, Proceedings of the Eighth International Conference on InP and Related Material, SchwabischGmund, Germany, 1996 (unpublished), WP-C 32.

${ }^{8}$ K. Knorr, A. Rumberg, M. Zorn, C. Meyne, T. Trepk, J.-T. Zettler, W. Richter, P. Kurpas, and M. Weyers, Proceedings of the Eighth International Conference on InP and Related Material, Schwabisch-Gmund, Germany, 1996 (unpublished).

${ }^{9}$ N. Dietz, U. Rossow, D. Aspnes, and K. J. Bachmann, J. Electron. Mater. 24, 1571 (1995)

${ }^{10}$ K. B. Ozanyan, P. J. Parbrook, M. Hopkinson, C. R. Whitehouse, Z. Sobiesierski, and D. I. Westwood, J. Appl. Phys. 82, 474 (1997).

${ }^{11}$ See, for example, M. Hopkinson and J. P. R. David, J. Cryst. Growth 175, 1033 (1997)

${ }^{12}$ J. P. Harbison, D. E. Aspnes, A. A. Studna, L. T. Florez, and M. K. Kelly, Appl. Phys. Lett. 52, 2046 (1988).

${ }^{13}$ F. Reinhardt, W. Richter, A. B. Muller, D. Gutsche, P. Kurpas, K. Ploska, K. C. Rose, and M. Zorn, J. Vac. Sci. Technol. B 11, 1427 (1993).

${ }^{14}$ J.-T. Zettler, J. Rumberg, K. Ploska, K. Stahrenberg, M. Pristovsek, W. Richter, M. Wassermeier, P. Schützendübe, J. Behrend, and L. Däweritz, Phys. Status Solidi A 52, 35 (1995).

${ }^{15}$ A. T. Payne, P. H. Fuoss, D. W. Kisker, and G. W. Stephenson, Phys. Rev. B 49, 14427 (1994). 